\title{
La atención a la diversidad: experiencia del Proyecto UNA Educación de Calidad, de la Universidad Nacional de Costa Rica
}

\section{Attention to Diversity: the Experience of UNA Quality Education Project, from the National University of Costa Rica}

\author{
Angélica Fontana Hernández ${ }^{1}$ \\ División de Educación Básica del \\ Centro de Investigación y Docencia en Educación, \\ Universidad Nacional \\ Heredia, Costa Rica \\ angelfontana@racsa.co.cr
}

Recibido 16 de julio de 2010 • Aceptado 31 de agosto de 2010

Resumen. Debido al marco jurídico actual, en los últimos años, el sistema educativo costarricense ha experimentado cambios significativos, tanto en los niveles internacional como nacional. Estos van desde la promulgación de la Ley 7600 Igualdad de Oportunidades para las Personas con Discapacidad en Costa Rica (Costa Rica, Asamblea Legislativa,1996) hasta el planteamiento de la Ley 8661 Convención sobre los Derechos Humanos de las Personas con Discapacidad (Costa Rica, Asamblea Legislativa, 2008).

Por lo anterior, el espacio temporal en el que emergió el Proyecto UNA Educación de Calidad, en 1998 y el contexto en el cual estaba inmerso el Sistema Educativo Nacional, particularmente, en el ámbito universitario, la propuesta pedagógica que se presenta da muestra de una transición entre el modelo rehabilitador y el modelo social, específicamente, dentro de cada uno de estos, el respectivo abordaje educativo, que abarca desde los procedimientos de la integración a los de la educación inclusiva.

Desde esta perspectiva, se presenta las vivencias y estrategias desplegadas desde el Proyecto UNA Educación de Calidad. El fin es proporcionar apoyo y seguimiento, en su formación profesional, a un grupo de la diversidad, constituido por los estudiantes con necesidades educativas matriculados en la Universidad Nacional. De esta forma, se pretenden hacer efectivos sus derechos de acceder a una educación superior de calidad, de acuerdo con sus características personales y sociales, y promover su permanencia e inserción socio-laboral.

Los retos que impone la atención a la diversidad en el contexto universitario son un desafío plasmado en el quehacer cotidiano del Proyecto UNA Educación de Calidad. Estas transformaciones se vislumbran en la constante reconstrucción de las estrategias utilizadas, al tener, como aspectos esenciales, el empleo de equipo tecnológico y programas de computación especializados. Estos les permitirán a los estudiantes acceder a la información y a la comunicación; la asesoría y la capacitación de los académicos y del personal administrativo; el establecimiento de estrategias de apoyo colaborativo entre los miembros de la comunidad universitaria, así como, la búsqueda de cooperación en el ámbito nacional e internacional para el desarrollo de nueva iniciativas.

\footnotetext{
Master en Pedagogía con énfasis en la diversidad de procesos educativos, Licenciada en Educación Especial con énfasis en Retardo Mental y Bachiller en Problemas de Aprendizaje de la Universidad Nacional de Costa Rica. Académica de la División de Educación Básica del Centro de Investigación y Docencia en Educación (CIDE) de la Universidad Nacional, Costa Rica y responsable del Proyecto UNA Educación de Calidad, que atiende estudiantes con necesidades educativas en su formación profesional. Con experiencia en educación primaria y en los servicios de educación especial. Tiene publicaciones en el campo de la pedagogía en el ámbito nacional e internacional.
} 
Palabras clave. Atención de las necesidades educativas, desarrollo integral, educación inclusiva.

Abstract. In the last years, the educational Costa Rican system has experienced significant changes, due to the legal framework, so much in the national and international levels, since the promulgation of the Law 7600 Equality of Opportunities for the Persons with Disability in Costa Rica (Costa Rica, Asamblea Legislativa,1996) to the proposal of the Law 8661 Convention on the Human rights of the Persons with Disability (Costa Rica. Asamblea Legislativa, 2008).

Due to the temporary space in which the UNA Quality Education Project emerged in 1998 and the context in which the Educational National System was immersed, particularly at the university level, the pedagogic offer reveals a transition between the rehabilitative model and the social model specifically, within each of these, the respective educational approach that includes procedures of integration and inclusive education.

From this perspective, experiences and strategies implemented at the UNA Quality Education Project are presented in order to provide support and follow-up in their vocational training to a group of students who present educational needs at the National University. Thus, it is pretended to make effective their rights to access a high quality education according to their personal and social characteristics, promoting their social and work insertion.

Challenges imposed by attention to diversity at the university context represent a defiance that is part of daily activities of the UNA Quality Education Project. These transformations are evident at the permanent redesign of strategies and the employment of technological equipment and specialized software. These changes allow students to access information and to communicate; the advising and training of academicians and the clerical staff, the establishment of collaborative support strategies between members of the university community, as well as the search of cooperation in the national and international area for the development of new initiatives.

Key words. Attention of the educational needs, integral development, inclusive education.

"Cuando los sueños se siembran en terreno fértil, germinan y crecen robustos, pero cuando estos sueños son cuidados con esmero y dedicación se recogen abundantes frutos"

Angélica Fontana

\section{A manera de introducción}

En la segunda mitad del siglo XX, la sociedad costarricense inicia un proceso de concienciación relacionada con la igualdad y equiparación de las oportunidades de las personas con discapacidad en diferentes ámbitos, particularmente, con el reconocimiento de sus derechos y su participación social.

Estos esfuerzos se ven influenciados por las declaraciones y convenios internacionales de este periodo: El Foro Mundial sobre Educación, con su proclama de una Educación para Todos (Organización de las Naciones Unidas para la Educación, la Ciencia y la Cultura [UNESCO], 1990), el cual busca la erradicación de la pobreza y la promoción del acceso a la educación como un derecho fundamental para todas las personas que históricamente se encuentran en desventaja social. Las Normas Uniformes sobre Equiparación de Oportunidades para las Personas con Discapacidad (Organización de la Naciones Unidas [ONU],1993), cuyo propósito era unificar disposiciones, reglas y conceptos en busca del bienestar y de la integración social de las personas con discapacidad, en su calidad de ciudadanos de sus respectivas sociedades, con los mismos derechos y obligaciones 
que las demás personas. La Conferencia Mundial sobre Necesidades Educativas Especiales: Acceso y Calidad (Organización de las Naciones Unidas para la Educación, la Ciencia y la Cultura [UNESCO], 1994), que orientaban el establecimiento de leyes y políticas nacionales que velaran por el cumplimiento de los derechos humanos de la personas, especialmente, en condición de discapacidad.

En nuestro país, en el año 1996, con la promulgación de la Ley 7600 Igualdad de Oportunidades para las Personas con Discapacidad (Costa Rica, Asamblea Legislativa, 1996), en el contexto educativo, se presenta una serie de cambios que se manifiestan en la organización de los centros escolares, las estrategias de apoyo, y la provisión de los materiales y recursos.

\section{El surgimiento del Proyecto UNA Educación de Calidad}

El ámbito universitario se ve permeado por las declaraciones y convenciones internaciones y por la legislación nacional vigente. Particularmente, en el año 1992, el Consejo Universitario solicita a la Vicerrectoría de Vida Estudiantil de la Universidad Nacional (UNA), mediante el acuerdo SCU 1893, la definición de las directrices institucionales para el establecimiento de las estrategias de apoyo que permitieran la integración de los estudiantes en condición de discapacidad en las distintas carreras de la Institución y, en el año 1994, se aprueba el Plan Global de atención del estudiante con discapacidad, con la participación del equipo multidisciplinario conformado por profesionales de distintas áreas de la universidad (Rodríguez et al., 1994).

En forma paralela a estos procesos institucionales, en el año 1998, como un iniciativa de la División de Educación Básica del Centro de Investigación en Docencia y Educación (CIDE), emerge el Proyecto UNA Educación de Calidad para todos los estudiantes de la Universidad Nacional Código: 010341, cuyo propósito consiste en ofrecer una respuesta a un grupo de estudiantes en condición de discapacidad, matriculados en las carreras de este centro.

Este proyecto, en congruencia con lo que establecía la legislación internacional y nacional en la primera mitad del siglo XX, así como con el modelo rehabilitador de atención de las personas con discapacidad que prevalecía en el sistema educativo nacional y, particularmente, con su abordaje pedagógico de la integración educativa, proporcionaba atención directa a los estudiantes en su formación profesional adaptando los materiales didácticos, proporcionando apoyo y seguimiento a los estudiante en su formación universitaria y asesorando a los académicos para que ajustaran sus intervenciones pedagógicas a las necesidades educativas especiales que presentaban los estudiantes y a los principios de justicia y solidaridad que regían el quehacer de la institución, según el Estatuto Orgánico (Universidad Nacional, 1993).

Es importante indicar que desde el abordaje pedagógico de la integración educativa, en el cual el Proyecto estaba inmerso, las principales estrategias empleadas estaban enfocadas en los apoyos educativos y en la adaptación de los medios de aprendizaje, con el fin de que cada estudiante pudiera acceder al currículo general (plan de estudio de cada carrera), donde las adecuaciones curriculares (no significativas) y de acceso al currículo, resultaban las formas más utilizadas.

Por tanto, el concepto de necesidades educativas especiales que se asume desde el Proyecto en este momento, se enfoca principalmente en los aspectos personales que presentan los estudiantes, las deficiencias sensoriales, físicas o dificultades en su aprendizaje y las carencias del entorno educativo para responder a estas diferencias individuales. Es decir, se consideraba que las necesidades educativas especiales se encuentran en estrecha relación con las ayudas pedagógicas o servicios de apoyo educativo que requieren los estudiantes en su formación profesional. 
Un aspecto particular es que el Proyecto contaba con la participación de estudiantes becados que realizaban horas asistentes y horas colaboración, quienes efectuaban diferentes funciones en el área administrativa y en la atención de las necesidades educativas especiales de los estudiantes que participaban del mismo. Entre estas funciones se destacaban la lectura en forma oral o el grabado de textos, documentos o libros de los diferentes cursos en que los estudiantes estaban matriculados; la orientación en el desplazamiento y la movilidad, como referente visual, dentro del campus Omar Dengo (sede central), en el salón de clase, en la búsqueda de referencias bibliográficas o bien en las visitas asignadas a los centros educativos o a otras instituciones públicas y privadas.

Además, mediante la iniciativa que nace de la carrera de Educación Especial de la División de Educación Básica del CIDE, se logró obtener financiamiento a través del Concurso Anual para la Distribución del Fondo Institucional de Desarrollo Académico (FIDA), en el año 2003, cuyo fin fue adquirir equipo tecnológico para facilitar el acceso a la información y a la comunicación de los estudiantes en condición de discapacidad sensorial (ceguera). Este equipo estaba conformado por computadoras, programas especializados como el lector de pantalla (JAWS), escáneres, impresoras Braille y grabadoras digitales. Se ubicó en la oficina del Proyecto y en el Centro de Información y Documentación en Educación, Niñez, Adolescencia y Familia (CIDENAF).

También, con el propósito de conformar una Sala de Apoyo Tecnológico Adaptado (SATA) que proporcionará servicios accesibles a los estudiantes (Herrera y Miranda, 2001), en este mismo periodo, en coordinación con la Dirección de la Biblioteca García Monge y la Comisión Institucional para la atención de los estudiantes con discapacidad, se puso a disposición un equipo de apoyo tecnológico, el cual incluía cinco computadoras, un escáner, una impresora Braille y un programa lector de pantalla (JAWS).

Un aspecto importante de destacar es que el equipo tecnológico adquirido facilitó el apoyo y el seguimiento a las necesidades educativas especiales que presentaban los estudiantes que participaban del proyecto; particularmente, se fortaleció la accesibilidad a la información y comunicación, porque se escaneaban textos, documentos y libros de los cursos para que los estudiantes pudieran acceder a estos mediante los programas lectores de pantalla, o bien, para ser transcritos al sistema Braille, y se ampliaba el material gráfico impreso según los requerimientos. De esta forma, desde el proyecto, se despliega una mayor cobertura de los apoyos proporcionados mediante la distribución de materiales didácticos en versiones audibles y digitales.

Si se reflexiona sobre las acciones realizadas desde el Proyecto UNA Educación de Calidad, en los años 1998 al 2001, se destacan los siguientes aspectos:

1. Se promueve en el contexto universitario una educación que tiene como valor fundamental el respeto a las diferencias humanas, por lo que posibilita a la población estudiantil entender y comprender las diferencias individuales.

2. Se brinda la oportunidad a los estudiantes, especialmente en condición de discapacidad, para que se incorporen y se desarrollen en igualdad de condiciones que el resto de sus compañeros.

3. Se fortalecen la didáctica universitaria porque los académicos adaptan la metodología, los materiales y recursos para responder a las necesidades educativas especiales de sus estudiantes.

4. Se promueve un trabajo en equipo entre los responsables del Proyecto UNA Educación de Calidad, los académicos y representantes de distintas instancias universitarias (Herrera, Miranda y Zúñiga, 1998; Herrera y Miranda, 2001). 
A partir de este momento, el Proyecto se constituye en la instancia universitaria que, desde su quehacer cotidiano, fortalece la igualdad y la equidad de oportunidades en el acceso a la educación superior de la población en condición de discapacidad.

\section{Crecimiento y despliegue de nuevas formas de apoyo}

A principios del siglo XXI emergen nuevas perspectivas acerca del universo, la vida y la supervivencia en el planeta; se discute sobre los derechos humanos y la inclusión social de los diferentes grupos que conforman la sociedad. Esto permitió generar espacios de reflexión y discusión en el ámbito internacional, donde los movimientos y foros sociales de los grupos que han sufrido discriminación y segregación, a través de la historia, por razones de etnia, género, posición social, discapacidad y preferencia sexual, tuvieron un papel determinante.

En este contex to de cambio, la UNA, como institución social y espacio de aproximación de la diversidad existente y creciente de la sociedad global (Fernández, 2001), y responsable de la formación universitaria de las futuras generaciones, se enfrenta al desafío de ofrecer respuestas curriculares a las diversas capacidades y necesidades presentes en la población estudiantil, así como, al de cumplir con su función social plasmada en el Plan Global Institucional 2004-2011 (Universidad Nacional, 2004 p. 36) que expresa:

(...) la Universidad contribuye a direccionar la sociedad hacia planos superiores de bienestar, equidad, sostenibilidad y libertad democrática, mediante nuevos paradigmas que permitan transformar y revalorar el desarrollo humano. Está comprometida con el desarrollo de toda la sociedad y en particular con la integración, potenciación y ampliación de oportunidades de los sectores sociales menos favorecidos o excluidos de los beneficios del desarrollo.

Por tanto, la educación y sus procesos de interacción e intercambio deben permitir, a los miembros de la comunidad universitaria, el reconocimiento de que el siglo XXI enfrenta transformaciones en el ámbito social, económico, político, ideológico y cultural, y que, con el impacto de la globalización, la diversidad se convierte en un valor fundamental para la convivencia pacífica y la supervivencia en este planeta y en el universo.

Por lo anterior, en los principales foros internacionales y nacionales sobre educación superior en el siglo XXI, se discuten temas relacionados con derechos humanos, emancipación de las minorías, ruptura de las fronteras étnicas y el reconocimiento del potencial humano que se genera en condiciones de diversidad (Meléndez, 2002), así como el establecimiento de estrategias institucionales que, con el fin de construir una sociedad más inclusiva, permitan el trabajo colaborativo y transdiciplinario entre las instituciones públicas y privadas.

Estos esfuerzos responden a la influencia de las declaraciones y convenios internacionales del momento. Entre estas se destacan las siguientes:

El Marco de Acción de Dakar. Educación para Todos: cumplir nuestros compromisos comunes (UNESCO, 2000) y la Declaración de Cochabamba, (UNESCO, 2001), en los cuales los Ministros de Educación de América Latina reiteran los compromisos asumidos anteriormente sobre la universalización de la educación básica, la alfabetización de la población de jóvenes y adultos, asimismo, conclusión de las reformas necesarias para mejorar la calidad y la eficiencia en la educación.

La Declaración Mesoamericana de Educación Inclusiva, en el año 2004 (Aguilar y Monge, 2008), cuyo propósito era fortalecer la cooperación internacional entre los países de la región 
para promover cambios sustantivos en las políticas y prácticas educativas, responder a las metas adoptadas en el Marco de Acción de Educación para Todos y sumar esfuerzos con el fin de lograr una mejor calidad de vida, sin discriminaciones de ningún tipo, reconociendo a la diversidad como fundamento para la convivencia social y garantizando la dignidad de la persona, sus derechos, su autodeterminación, su contribución a la vida comunitaria y su pleno acceso a los bienes sociales.

La Declaración de Montreal sobre Discapacidad Intelectual en el año 2004, promovida por la Organización Mundial de la Salud (OMS, 2004) y la Organización Panamericana de la Salud (OPS), con la finalidad de promover las medidas legislativas, jurídicas, administrativas y educativas necesarias para lograr la plena inclusión social de las personas con discapacidades intelectuales.

Con la experiencia acumulada en el Proyecto UNA Educación de Calidad a lo largo de los años y los espacios de intercambio, reflexión y aprendizajes construidos en el ámbito universitario con los estudiantes en condición de discapacidad, académicos, administrativos, estudiantes asistentes y de horas colaboración, se generan nuevas iniciativas que responden a las demandas de este nuevo siglo.

En los años 2002 y 2003 las personas responsables del proyecto, de la Comisión Institucional para la atención de los estudiantes con discapacidad y de la Vicerrectoría de Vida Estudiantil organizaron una serie de talleres, con diferentes representantes de las distintas instancias universitarias, para formular las políticas institucionales en materia de discapacidad. El fruto de este esfuerzo culminó con la aprobación, por parte del Consejo Universitario en el año 2005, de estas Políticas Institucionales, en las cuales, se destaca que “(...) la actividad de la UNA consiste en coadyuvar a la promoción de los sectores menos favorecidos mediante la puesta en práctica de políticas de inclusión e igualdad de oportunidades" (Universidad Nacional, 2005, p. 1).

Desde la dinámica cotidiana que se despliega en el proyecto en este momento y con el fin de responder a la legislación y convenios vigentes, se destaca la apertura, por parte de las académicas responsables, de ampliar su cobertura a todos los estudiantes de la Institución que presentan necesidades educativas en su formación universitaria. Lo anterior, debido a que se asume una concepción más amplia y flexible acerca de las necesidades educativas, se consideran las diferentes conceptuaciones presentadas en los foros y declaraciones mundiales organizadas por la ONU y la UNESCO, en las cuales se toman en cuenta, tanto aspectos personales de los estudiantes como socioculturales, con el fin de garantizar una educación de calidad para todos los grupos que conforman la comunidad universitaria, sin ningún tipo de discriminación ni exclusión.

Por lo antecedente, las necesidades educativas se definen como las condiciones de aprendizaje que surgen de la interacción entre las diferencias individuales de los estudiantes y el entorno social que presenta barreras actitudinales, físicas, arquitectónicas, pedagógicas y culturales (Fontana, Espinoza y León, 2009).

Las necesidades educativas percibidas y valoradas desde este nuevo enfoque de atención a la diversidad e inclusión social generan un cambio de concepción en el sentido de que no solo los estudiantes en condición de discapacidad pueden presentar situaciones específicas en su formación profesional, pues se considera que estas pueden surgir tanto por factores personales como socioculturales.

Desde esta perspectiva, el término especial está superado, porque hacía alusión a que la necesidad educativa surgía solamente de la condición de discapacidad que presentaba el estudiante $y$, en este contexto, cualquier estudiante, según sus diferencias personales y sociales, puede presentar necesidades educativas.

Al respecto, el Centro Nacional de Recursos para la Educación Inclusiva (CENAREC, 2005) indica que las necesidades educativas pueden surgir por múltiples causas. Estas pueden ser por 
condiciones personales de los estudiantes, tales como deficiencias físicas, sensoriales o mentales, por su forma particular de aprender o bien por la interrelación de sus particularidades con su entorno social: familia, escuela o comunidad, que limitan su desarrollo y aprendizaje. También, se pueden derivar por los estilos de enseñanza que no consideran las condiciones de aprendizaje ni las adaptaciones que requieren los estudiantes, así como por la falta de productos de apoyo (recursos tecnológicos y ayudas técnicas).

Por otra parte, un aspecto esencial de destacar consiste en que las necesidades educativas presentan dos características significativas (Sánchez y Torres, 2002). La primera, su carácter interactivo, es decir, la necesidad se define en relación con el contexto social en que se desenvuelve el estudiante y no solo dependen de sus condiciones personales; la segunda, la dimensión relativa de la necesidad, la cual está determinada por el tiempo y el espacio en que se encuentra, por tanto, no pueden tener un carácter definitivo o permanente.

A partir de este momento, desde la dinámica cotidiana, el Proyecto UNA Educación de Calidad, inicia un periodo de transición entre el modelo clínico, prevaleciente en el sistema educativo, y el modelo social emergente. Y dentro de cada uno de ellos, el respectivo abordaje educativo, que va desde los procedimientos de la educación integradora a los de la educación inclusiva.

Por lo anterior, el proyecto experimenta un crecimiento significativo en la cantidad de estudiantes solicitantes de los servicios ofrecidos, pasando de 25 en el año 2005 a 96 estudiantes activos para el 2010, matriculados en distintas carreras de las diversas unidades académicas de la Universidad Nacional, entre estas: Educación Preescolar, Educación Especial, Pedagogía en I y II Ciclos, Relaciones Internacionales, Psicología, Orientación, Educación Musical, Enseñanza del Inglés, Bibliotecología, Enseñanza de la Religión, Enseñanza de la Ciencias, Enseñanza del Francés, Arte y Comunicación, Economía, Ciencias Forestales, Administración de Oficinas, Relaciones Internacionales, Filosofía, Historia, Ciencias Geografías, Sociología, Administración Empresarial, Sociología entre otras (Fontana, Vargas y Holst, 2009).

Las personas responsables del Proyecto propician la coordinación sostenida a lo largo de los dos ciclos lectivos con las unidades académicas y los académicos que atienden estudiantes con discapacidad u otra condición matriculados en sus cursos. De esta forma, se promueve una debida atención a las necesidades educativas de los estudiantes y la capacitación a los académicos. Particularmente, se mantiene comunicación en forma escrita, vía telefónica y en forma virtual (Internet), así como, se proporciona, mediante folletos y desplegables, la divulgación de información sobre la atención de las necesidades educativas, en especial a aquellas relacionadas con condición de discapacidad.

Desde el II ciclo del año 2006, se implementaron dos acciones nuevas que permitieron una mayor comunicación y organización con las unidades académicas, una de estas fue la visita a las asambleas de académicos para presentar las funciones que se realizan en el Proyecto y realizar diferentes estrategias de sensibilización y capacitación acerca de la atención de las necesidades educativas en condición de discapacidad u otra situación particular. Posteriormente, se envía una nota para que se designe a un funcionario de enlace entre la onidad académica y el Proyecto. Estas acciones resultaron muy exitosas; pero por el poco personal de apoyo del Proyecto, solo se ha podido visitar la mitad de las unidades académicas en que están inscritos la totalidad de los estudiantes. Es importante indicar que se ha dado prioridad a las unidades académicas que tienen estudiantes con discapacidad física, sensorial (visual y auditiva) y cognitiva (Fontana y Vargas, 2007).

La atención directa de los estudiantes sigue siendo una acción prioritaria en el quehacer del Proyecto, por esto en el año 2007 se logra gestionar, con el actual Vicerrector de Desarrollo, la remodelación del aula 802 del CIDE, para que el Proyecto contara con un espacio acondicionado que 
permitiera atender de la mejor manera a los estudiantes, especialmente, en condición de discapacidad. No es sino hasta el año 2008, cuando se logra concretar la iniciativa y, a partir de este momento, se trabaja en un espacio propio, más accesible y acogedor (Fontana, Vargas y Holst, 2008).

También, en el año 2008, se logró financiamiento a través del Concurso Anual para la Distribución del Fondo Estatal de la Educación Superior (FEES), con el fin de adquirir mayor equipo tiflotécnológico, el cual estaba conformado por computadoras portátiles, nuevos programas computaciones especializados (lectores de pantalla-JAWS, traductores de audio-Dragon Speaking, Magnificadores de imágenes-Magic, reproductores de voz-Voice Editor, traductor de música al sistema Braille-Goodfeel Braille Music Tralation, traductor de texto al sistema Braille-Duxbury), horno Thermoform para imprimir materiales en relieve, cámara de video, protector multimedia, amplificador de sonido, grabadoras digitales entre otros, los que se ubicaron, principalmente, en la nuevas instalaciones del Proyecto, en la Sala de Equipo Tecnológico Adaptado (SATA) de la Biblioteca Joaquín García Monge y en otras centros de información y documentación de las facultades de la Institución, así como, en el centro de cómputo de la residencias estudiantiles.

Es importante indicar que la adquisión de este equipo ha permitido facilitar y diversificar las formas de apoyo y seguimiento a las necesidades educativas de los estudiantes que participan en el Proyecto. Entre las principales acciones se destacan las siguientes:

a. Préstamo de equipo tecnológico, de productos de apoyo y de accesorios, tales como, computadoras de escritorio o portátiles con programas especializados (lectores de pantalla-JAWS, traductores de audio- Dragon Speaking, Magnificadores de imágenes-Magic, reproductores de voz-Voice Editor) y grabadoras periodísticas digitales con salida USB o sin ella.

b. Impresiones de textos y documentos en el sistema Braille y en tinta.

c. Escaneo de textos, documentos y libros para que los estudiantes puedan acceder a estos mediante el lector de pantalla.

d. Ampliación de material impreso.

e. Impresión de materiales en relieve, particularmente, figuras, mapas gráficos y partituras de música.

También se continúa empleando y fortaleciendo, como una estrategia acertada, el apoyo de estudiantes becados que realizan horas asistentes y colaboración que coadyuvan en el quehacer cotidiano del Proyecto. Actualmente, se cuenta con un promedio de 25 estudiantes por ciclo lectivo en diferentes labores en el área administrativa y en la atención directa de las necesidades educativas de los estudiantes, principalmente, apoyan en la adaptación de los materiales y recursos didácticos; en el escaneo de texto, documentos y libros; en las ampliaciones e impresiones en relieve de cuadros, tablas, figuras, gráficos y partituras musicales, según los requerimientos. Además, se facilita el acceso a la Internet para la búsqueda de información y la comunicación mediante el correo electrónico.

Otra forma de apoyo exitosa implementada recientemente consiste en el trabajo entre pares con estudiantes con deficiencia visual y que requieren atención individual para desplegar mayores habilidades y destrezas en el uso de los programas computacionales especializados, el desplazamiento y la movilidad en el campus Omar Dengo y en las actividades de vida independiente. Esto ha permitido que se fortalezcan los valores de la solidaridad, el respeto y la amistad entre los estudiantes y las personas que participan del Proyecto UNA Educación de Calidad, así como el concepto de comunidad que aprende cada día y se reconstruye con las experiencias personales y sociales de cada uno de sus miembros (Flores, L. E, Flores, G., Jiménez, Madrigal y Perearnau 2009). 
Otra actividad fundamental del Proyecto es la asesoría y capacitación en forma individual o en grupo a los académicos y a los administrativos de las unidades académicas, con el fin de promover una atención educativa de calidad, según las necesidades educativas de los estudiantes. Estos asesoramientos se desarrollan empleando el intercambio de ideas, de conocimientos y de experiencias relacionadas con la temática, específicamente, se revisa la documentación que contiene el expediente del estudiante, se presentan las características personales y sociales, los posibles ajustes curriculares y apoyos que requiera en su formación profesional, considerando la naturaleza del curso (teórico, teórico-práctico, laboratorio o de ejecución) y las recomendaciones de los especialistas. Durante el año 2009 se efectuaron un promedio de 50 sesiones de asesoría con los académicos y administrativos de diferente unidades académicas. Estas tuvieron, en su mayoría, una incidencia positiva en las futuras intervenciones pedagógicas, así como en la comunicación e interacción con los estudiantes (Fontana y otros, 2009).

También se propicia la coordinación con otras instancias o departamentos de la Institución, para tramitar las solicitudes de los estudiantes que tienen relación con la atención de sus necesidades educativas o al reconocimiento de sus derechos en el ámbito universitario. Para ello, se hacen gestiones con la Comisión Institucional de Atención al Estudiante con Discapacidad, o bien con el Departamento de Planeamiento Espacial, Departamento de Orientación y Psicología, Departamento de Bienestar Estudiantil, Departamento de Salud, Unidad de Admisión, Departamento de Registro, entre otros.

Entre las principales acciones de coordinación se destacan las siguientes:

En los años 2004 a 2005, el Proyecto coordina con los responsables del Área de Planeamiento Espacial de la Institución para la realización de un diagnóstico del campus Omar Dengo (sede central), cuyo fin es determinar las barreras arquitectónicas y físicas, así como establecer los ajustes requeridos (rampas, barandas de apoyo, señalización entre otros) para generar mayores condiciones de accesibilidad. No es hasta el año 2007, cuando se construyen unas rampas con barandas de apoyo en el trayecto de las residencias estudiantiles hacia las oficinas del Proyecto, así como, en otros sectores del campus, con lo cual se facilita el acceso a los estudiantes en condición de discapacidad física y a las personas que ingresan a la Institución. No obstante, el acceso al espacio físico y a la infraestructura es un tema pendiente que requiere mayor atención de parte de las autoridades universitarias.

Desde los años 2006, 2007 y 2008, las responsables del Proyecto, en coordinación con académicas de la carrera de Educación Especial del CIDE y de otras instancias universitarias, formulan el Programa UNA Diversa como la instancia institucional encargada de organizar, asesorar y ejecutar las acciones tendientes para promover la igualdad y la equidad en el acceso a las oportunidades, bienes y servicios que brinda la UNA a los grupos que conforman la diversidad de la comunidad universitaria, así como de coordinar con otros programas y proyectos a nivel institucional, nacional e internacional. Esta iniciativa está en espera de la obtención de recursos financieros que permitan su implementación.

En el año 2007 y 2008 se proporciona asesoría al Departamento de Bienestar Estudiantil y al personal del sistema bibliotecario de la Universidad, en particular, sobre estrategias de atención al usuario en condición de discapacidad y la definición de los ajustes del entorno laboral para generar condiciones de accesibilidad.

En los años 2007, respondiendo a una resolución de la Defensoría de los Habitantes, en la cual se recomienda a la Universidad la elaboración de una normativa para la aplicación de las adecuaciones en el ámbito universitario, el señor Rector, Dr. Olmán Segura, forma una comisión institucional para que redacte este documento. La misma, está conformada por representantes del 
Proyecto UNA Educación de Calidad, de la Asesoría Legal, de la carrera de Educación Especial del Centro de Investigación en Docencia y Educación (CIDE), de la Vicerrectoría de Vida Estudiantil, de Diseño Curricular y de otras instancias universitarias. Esta comisión redacta el borrador del Reglamento institucional para la garantía de los derechos y la equiparación de oportunidades en el acceso a los bienes y servicios que proporciona la Institución, con el propósito de promover el desarrollo integral de todos los miembros de la comunidad educativa en condiciones de equidad e igualdad. Actualmente, esta iniciativa está en revisión y en espera de su aprobación en el Consejo Universitario.

También se colabora con la Vicerrectoría de Vida Estudiantil en la búsqueda de recursos humanos de apoyo para los estudiantes, especialmente, con deficiencia auditiva que requieren de intérpretes para acceder a la información y a la comunicación en su formación profesional. Además, se apoya a la Unidad de Admisión en la definición de los ajustes de accesibilidad para la Prueba de Admisión a la Institución y en la aplicación de la misma en los periodos respectivos.

Otra actividad que genera resultados significativos es la realización de talleres y convivíos entre los estudiantes que participan del Proyecto, particularmente, porque permite un espacio de esparcimiento, recreación y reflexión sobre las experiencias vividas tanto en el ámbito personal como en el académico; asimismo, se valora la calidad del apoyo y el seguimiento que se proporciona. Es importante resaltar que la información recabada en estas actividades es un insumo valioso para reformular los objetivos que se plantea alcanzar en el plan de trabajo anual (Fontana, Vargas, M., Holst, Vargas, A. y Sánchez, 2008).

Si bien la perspectiva teórica que sustenta el abordaje pedagógico de la integración educativa proporciona una valoración más positiva de las personas con discapacidad, como sujetos con derechos individuales y sociales, en sus prácticas y particularmente en la educativa, ha generado cambios significativos en su organización y en la didáctica (Aguilar, 2004); aún prevalecen, principalmente en el ámbito universitario, distintas formas de segregación, de discriminación y de exclusión, las cuales se reflejan en los ámbitos social y laboral.

\section{Retos actuales}

El siglo XXI se vislumbra como la era de los cambios continuos y profundos, abundantes retos y oportunidades. Según, Michalski y Stevens (1999), cada vez es más evidente el crecimiento -y así continuará- de la diversidad y de la complejidad de las exigencias impuestas a los sistemas de educación y capacitación de los países.

En este contexto social de cambio, emerge el modelo social como una tendencia que responde a las aspiraciones emancipadoras de los distintos grupos sociales. En esta tendencia, el movimiento de vida independiente de las personas con discapacidad tiene eco. A partir de este enfoque, la concepción de la discapacidad cambia y se centra la atención en el entorno físico-social, el cual presenta barreras que limitan el disfrute de los Derechos Humanos.

Por lo anterior, en el año 2004, la Organización Panamericana de la Salud (OPS) expresa que la discapacidad es una condición humana que puede ser de origen multicausal, ya sea por una circunstancia genética, de pobreza, desnutrición o inadecuados hábitos de higiene, enfermedad o accidente laboral, deportivo, recreativo y de tránsito, así como, de contaminación ambiental, violencia política, social y estructural, y que ninguna persona puede ser discriminada por razón, ni por género, estrato social, nivel educativo, preferencia sexual o bien por pertenecer a una etnia o religión. 
En concordancia con lo expresado por la OPS, la ONU aprueba en el año 2006 la Convención sobre los Derechos Humanos de las Personas con Discapacidad, el primer tratado internacional acerca de los derechos humanos en este siglo XXI. Su propósito es promover, proteger y asegurar el goce pleno y en condiciones de equidad de todos los derechos humanos y libertades fundamentales para todas las personas con discapacidad, así como promover el respeto a su dignidad humana.

Por tanto, en esta convención, la discapacidad se concibe como el resultado de la interacción entre las personas que presentan deficiencias y las barreras en las actitudes de la sociedad y del entorno, las cuales evitan su participación plena y efectiva en igualdad de condiciones (ONU, 2008). Por tanto, al definirse la discapacidad desde este enfoque interactivo entre las condiciones individuales de las personas y el contexto social, se asume, entonces, a la atención de sus necesidades, un valor relativo, el cual debe considerarse en el abordaje pedagógico.

Si se reflexiona acerca de las acciones realizadas por el Proyecto UNA Educación de Calidad del año 2000 al 2009 y se retoma las recientes declaraciones y convenciones se presentan los siguientes retos:

1. Desplegar diferentes acciones de concienciación (talleres, capacitaciones, grupos de discusión, pasantías) que permita a los miembros de la comunidad universitaria el reconocimiento de la diversidad como un valor fundamental en las relaciones humanas y en la formación universitaria.

2. Establecer una estrategia de coordinación entre el Proyecto UNA Educación de Calidad y las distintas instancias universitarias con el fin de definir acciones de cooperación en la atención de las necesidades educativas que presentan los estudiantes desde una perspectiva de atención a la diversidad e inclusión social.

3. Desarrollar diferentes acciones de coordinación entre el Proyecto UNA Educación de Calidad y las distintas instancias universitarias que permitan a los estudiantes una participación plena en todas las actividades de la comunidad universitaria (culturales, recreativas, deportivas, académicas o laborales) que incidan en su desarrollo personal, emocional, académico, y profesional, así como en la toma de decisiones, para asegurar el goce de sus derechos como miembros activos.

4. Desplegar estrategias de coordinación entre el Proyecto UNA Educación de Calidad, la carrera de Educación Especial y otras carreras del CIDE, así como otras instancias institucionales para fortalecer la realización de diferentes actividades y proyectos conjuntos desde una perspectiva de atención a la diversidad e inclusión social (trabajos finales de graduación, proyectos de investigación, giras educativas entre otras).

5. Establecer estrategias de cooperación con instituciones públicas, privadas u organizaciones no gubernamentales en el ámbito nacional e internacional para la implementación del Programa UNA-Diversa en la UNA.

Después de realizar un análisis retrospectivo sobre las diferentes etapas evolutivas, en las cuales ha transcurrido el Proyecto UNA Educación de Calidad, de la Universidad Nacional, se puede afirmar que, desde su surgimiento hasta su consolidación, ha desarrollado una actividad permanente en el ámbito universitario; las diversas iniciativas desarrolladas en sus diferentes momentos han marcado un precedente en la atención de la diversidad, fortaleciendo la igualdad y la equidad en el acceso a los bienes y servicios prestados por la Institución y ha brinda o sin o sin do las estrategias necesarias para la permanencia de la población estudiantil y su inclusión social (Fontana, 2009). 


\section{Referencias bibliográficas}

Costa Rica. Asamblea Legislativa. (1996). Ley 7600. Igualdad de Oportunidades para la Personas con Discapacidad. San José, Costa Rica: Imprenta Nacional.

Aguilar, G. (2004, Julio). Del exterminio a la educación inclusiva: Una visión desde la discapacidad. Conferencia presentada en el V Congreso Educativo Internacional: De la educación tradicional a la educación inclusiva. Heredia: Universidad Interamericana de Costa Rica.

Aguilar, G. y Monge, G. (2008) La Educación Especial en Costa Rica: antecedentes, evolución, nuevas tendencias y desafíos. San José: Ministerio de Educación Pública. Versión preliminar sin publicar.

Centro Nacional de Recursos para la Inclusión Educativa [CENAREC]. (2005). Adecuaciones de Acceso al Currículo. Inducción a Equipos de Investigación. San José: CENAREC. Material sin publicar.

Costa Rica. Asamblea Legislativa. (2008, Setiembre 29). Convención sobre los Derechos Humanos de las Personas con Discapacidad (Ley $N^{\circ} 8661$ ). Diaio Oficial La Gaceta $N^{\circ} 187$ San José: Imprenta Nacional.

Fernández, M. (2001). Educar en tiempos inciertos. Madrid: Ediciones Morata.

Fontana, A. (2009). Retos de la atención a la diversidad: el caso de la Universidad Nacional de Costa Rica. La Gaceta Visón Docente Con-Ciencia del Centro de Estudios Universitarios Arkos de Puerto Vallarta, México No 51 p.30-40. Recuperado el 15 de setiembre del 2010, de http:// www.ceuarkos.com/vision docente/revistas/51/retos.pdf

Fontana, A., Espinoza, A. y León, H. (2009). Implementación de las adecuaciones de acceso en las instituciones educativas costarricenses. San José: CENAREC.

Fontana, A. y Vargas, M. (2007). Informe anual del Proyecto UNA Educación de Calidad. Heredia: Vicerrectoría Académica, Universidad Nacional. Material sin publicar.

Fontana, A., Vargas, M. y Holst, B. (2008). Informe anual del Proyecto UNA Educación de Calidad. Heredia: Vicerrectoría Académica, Universidad Nacional. Material sin publicar.

Fontana. A, Vargas, M., Holst, B., Vargas, A. y Sánchez, M. (2008). Guía Metodológica: Encuentro de estudiantes del Proyecto UNA Educación de Calidad. Heredia: Centro de Investigación en Docencia y Educación, Universidad Nacional. Material sin publicar.

Flores, L. E., Flores, G., Jiménez, R., Madrigal, J. C. y Perearnau, M. A. (2009). Comunidad aprendiente. San José: ARTEMUSA Editorial.

Herrera, A., Miranda, G. y Zúñiga, E. (1998). Informe anual del Proyecto UNA Educación de Calidad. Heredia: Vicerrectoría Académica, Universidad Nacional. Material sin publicar. 
Herrera. A. y Miranda, G. (2001). Informe anual del Proyecto UNA Educación de Calidad. Heredia: Vicerrectoría Académica, Universidad Nacional. Material sin publicar.

Meléndez, L. (2002). La inclusión escolar del alumno con discapacidad intelectual. Bogotá: Creamos alternativas GLARP-IIPP.

Michalshi, W. y Stevens, B. (1999). Economía sociedad y capacitación: Retos para la educación y la capacitación en el siglo XXI. México: Noriega Editores.

Organización de las Naciones Unidas para la Educación, la Ciencia y la Cultura [UNESCO]. (1990, 5-9 de Mazo). Declaración Mundial sobre educación para todos "Satisfacción de las necesidades básica de aprendizaje”. Celebtado en Jomtien, Tailandia. Recuperado de http://www. oei.es/efa2000jomtien.htm

Organización de las Naciones Unidas [ONU]. (1993, Diciembre 20). Normas Uniformes sobre la Igualdad de Oportunidades para personas con discapacidad. Nueva York. Recuperado de http://www.cnree.go.cr/en/biblioteca-legislacion/normas-uniformes-sobre-la-igualdad-deoportunidades-para-personas-con-discapa.html

Organización de las Naciones Unidas [ONU]. (2008, Noviembre 3). Convención sobre los Derechos de las Personas con Discapacidad. Recuperado de http://www.un.org/spanish/disabilities/ pdf/SpanishCPRD.CSP.2008.1.pdf

Organización de las Naciones Unidas para la Educación, la Ciencia y la Cultura [UNESCO]. (1994, Junio 10.) Conferencia Mundial sobre Necesidades Educativas Especiales: acceso y calidad. Salamanca, España. Recuperado de http://paidos.rediris.es/genysi/recursos/doc/leyes/dec_sal. $\underline{\text { htm }}$

Organización de las Naciones Unidas para la Educación la Ciencia y la Cultura [UNESCO]. (2000, Abril 26-28). Marco de Acción de Dakar. Educación para Todos: cumplir nuestros compromisos comunes. Dakar, Senegal. Recuperado de http://www.unesco.org/education/efa/ ed for all/dakfram spa.shtml

Organización de las Naciones Unidas para la Educación la Ciencia y la Cultura [UNESCO]. (2001, Marzo 6). Declaración de Cochabamba. Bolivia, Cochabamba. Recuperado de http://www. ocimed.gob.pe/documentos_obs/compro_marco/cochabamba.pdf

Organización Mundial de la Salud [OMS]. (2004). La Declaración de Montreal sobre la Discapacidad Intelectual. Montreal: Organización Mundial de la Salud.

Rodríguez, M., Jiménez, F., Benavides, Y., Miranda, G., Torres, A., Vargas, E., Zúñiga, E., Cárdenas, F., Chacón, E. y Delgado, R. (1994). Plan Global de atención del estudiante con discapacidad. Heredia: Universidad Nacional. 
Sánchez, A. y Torres, J. (2002). Educación Especial. Centros educativos y profesores ante la diversidad. Madrid: Ediciones Pirámide.

Universidad Nacional. (1993). Estatuto Orgánico. Heredia: Departamento de Publicaciones UNA.

Universidad Nacional. (2004). Plan Global Institucional 2004-2011. Heredia: Departamento de Publicaciones UNA.

Universidad Nacional. (2005). Políticas Institucionales. Heredia: Gaceta N ${ }^{\circ} 1$ acuerdo del SUC2369-2004 Departamento de Publicaciones de la UNA.

Vargas, M., Fontana. A y Holst, B. (2008). Memoria: Inauguración de las instalaciones del Proyecto UNA Educación de Calidad y Foro: Educación Superior y Convención sobre los Derechos Humanos de las personas con discapacidad. Heredia: Centro de Investigación en Docencia y Educación, Universidad Nacional. Material sin publicar. 